\title{
Réseaux bancaires et banques en réseau en Bretagne
} R. Séchet

Citer ce document / Cite this document :

Séchet R. Réseaux bancaires et banques en réseau en Bretagne. In: Norois, n¹76, Octobre-Décembre 1997. pp. 605-622; doi : 10.3406/noroi.1997.6825

http://www.persee.fr/doc/noroi_0029-182x_1997_num_176_1_6825

Document généré le 12/06/2016 
Norois, 1997, Poitiers, $t .44, \mathrm{n}^{\circ} 176$, p. 605-622.

\title{
NOTES ET CHRONIQUE DE BRETAGNE
}

\author{
RÉSEAUX BANCAIRES \\ ET BANQUES EN RÉSEAUX EN BRETAGNE
}

\author{
par Raymonde SÉCHET \\ UMR 6590 ESO \\ Département de géographie \\ Université Rennes 2
}

L'activité bancaire a profondément évolué depuis la loi Debré de 1966 qui a autorisé l'entrée des banques françaises dans l'ère de la concurrence et de la diversification. A une première période de "bancarisation » marquée par l'extension des réseaux d'agences fait suite une période de consolidation qui, même si elle a débuté au milieu des années 1970 , s'est véritablement exprimée avec la loi bancaire de 1984 destinée à favoriser la modernisation bancaire et qui reconnaît le principe d'universalité. Cette seconde période est caractérisée par de subtils mouvements internes aux entreprises bancaires, mouvements qui aboutissent à une structure organisationnelle et territoriale complexe. La forte implantation régionale des établissements bancaires mutualistes donne tout son intérêt à l'étude des réseaux bancaires en Bretagne et de l'articulation entre les stratégies de recompositions des organisations bancaires et l'armature urbaine régionale.

L'étude présentée ici a été réalisée dans le cadre d'un programme de recherche du PIR-Villes sur "Réseaux de villes et villes en réseaux " financée par la DATAR. Elle porte plus particulièrement sur les banques adhérant à l'Association française des banques (AFB), et notamment les «trois grandes » que sont la BNP, le Crédit Lyonnais, la Société générale, et sur les banques mutualistes et coopératives (Caisses de crédit agricole, Caisses de crédit maritime mutuel, Caisses de crédit mutuel, Banques populaires). Elle exclut donc les services financiers de la Poste, les Caisses d'épargne et de prévoyance «Ecureuil », ainsi que les établissements de crédit qui ne sont pas autorisés à collecter des dépôts à vue. Le champ géographique est la Bretagne partiellement élargie à la Loire-Atlantique dans la mesure où la Banque populaire Bretagne-Atlantique qui couvre le Sud-Finistère, le Morbihan et la Loire-Atlantique a son siège à Nantes. La première partie du texte portera sur le maillage du territoire par les banques. Nous étudierons dans une seconde partie l'organisation interne des appareils et les recompositions qui aboutissent à une redéfinition des rôles de chacun des niveaux hiérarchiques et des modalités d'articulation entre ceux-ci.

\section{I. - AU CONTACT DU CLIENT, LE MAILLAGE DU TERRITOIRE PAR LES AGENCES BANCAIRES}

La banque est par nature une activité urbanophile et de proximité. La contrainte de la relation personnelle est en effet telle que les entreprises bancaires doivent se localiser à proximité de leurs clients. L'occupation de l'espace par chacune des 
banques et sa dynamique sont le reflet de l'accroissement de la demande inhérente aux transformations de la société ; de l'émergence d'un contexte de plus en plus concurrentiel qui a imposé de véritables stratégies spatiales pour capter les clients ; de l'histoire de chacune des entreprises bancaires, histoire qui tient à des «vocations » c'est-à-dire à l'existence de clientèles préférentielles, mais aussi à l'évolution vers toujours plus de libéralisme du cadre législatif organisant le système bancaire national.

\section{A) BANQUES ET RÉSEAUX URBAINS}

Le nombre de bureaux et d'agences ainsi que le nombre d'enseignes bancaires présentes dessinent clairement la hiérarchie urbaine bretonne (fig. l).

- Au premier niveau se placent Rennes qui compte 97 agences (ou bureaux) pour 29 enseignes et Brest avec 68 agences pour 20 enseignes.

- A un second niveau, on trouve Lorient (42 agences ou bureaux et 23 enseignes) et Quimper ( 41 agences et 20 enseignes). Les trois villes qui viennent ensuite apparaissent en retrait, surtout parce que le nombre d'enseignes présentes est nettement plus faible. Il s'agit de Vannes (32 agences et 13 enseignes), SaintBrieuc (30 agences et 13 enseignes), Saint-Malo (33 agences et 11 enseignes).

- Vient ensuite tout un ensemble de villes comptant entre 10000 et 20000 habitants dont Vitré ( 9 agences et 7 enseignes), Dinan ( 10 agences et 8 enseignes), Concarneau ( 11 agences et 9 enseignes), Pontivy ( 10 agences et 9 enseignes).

- Aux deux derniers niveaux se trouvent les petites villes comme Bain-de-Bretagne et Combourg qui comptent chacune 4 agences et enseignes ou comme La Guerche de Bretagne ( 5 agences et enseignes) et de nombreuses communes rurales qui disposent d'une ou deux agences et enseignes.

Cette hiérarchie qui est dans ses grandes lignes le reflet du réseau urbain breton souligne clairement la forte densité bancaire du littoral et, à l'inverse, une desserte beaucoup plus faible du Centre-Bretagne où, à quelques exceptions près comme Loudéac, Pontivy, Malestroit, Guer, Carhaix, l'offre est souvent réduite à une ou deux agences et enseignes (auxquelles s'ajoute la Poste). La forte présence bancaire sur le littoral ne s'explique pas totalement par la répartition de la population bretonne. Des stations balnéaires font figure de résidus positifs, comme si la distinction spatiale avait attiré les banquiers : Saint-Cast et Sarzeau comptent chacune 5 agences et enseignes pour respectivement 3093 et 4972 habitants ; Saint-QuayPortrieux et Erquy comptent chacune 6 agences et enseignes pour respectivement 3018 et 3568 habitants ; avec seulement 4243 habitants, Carnac dispose de 7 agences et enseignes bancaires alors que Quiberon compte 8 agences et enseignes pour 4623 habitants.

Les couronnes périurbaines apparaissent par contre comme des résidus négatifs : avec respectivement 4 361, 7 013, 5566 habitants en 1990, Acigné, Betton, Pacé ne disposent que de deux enseignes et agences. En fait la diversification de l'offre va largement de pair avec l'ancienneté du processus d'urbanisation. Ainsi, toujours dans l'agglomération rennaise, Chantepie (5 898 habitants), Saint-Grégoire (5 809 habitants) et Bruz ( 8114 habitants) disposent chacune de quatre agences et enseignes. La présence bancaire est plus forte encore à Cesson-Sévigné ( 7 agences et enseignes pour 12708 habitants). Par ailleurs, la faible desserte des couronnes périurbaines en guichets bancaires n'exclut pas une forte présence de l'activité bancaire si l'on prend en compte le glissement vers l'extérieur des villes de fonctions centrales en quête d'espace et de facilité d'accès et de stationnement. C'est le cas 


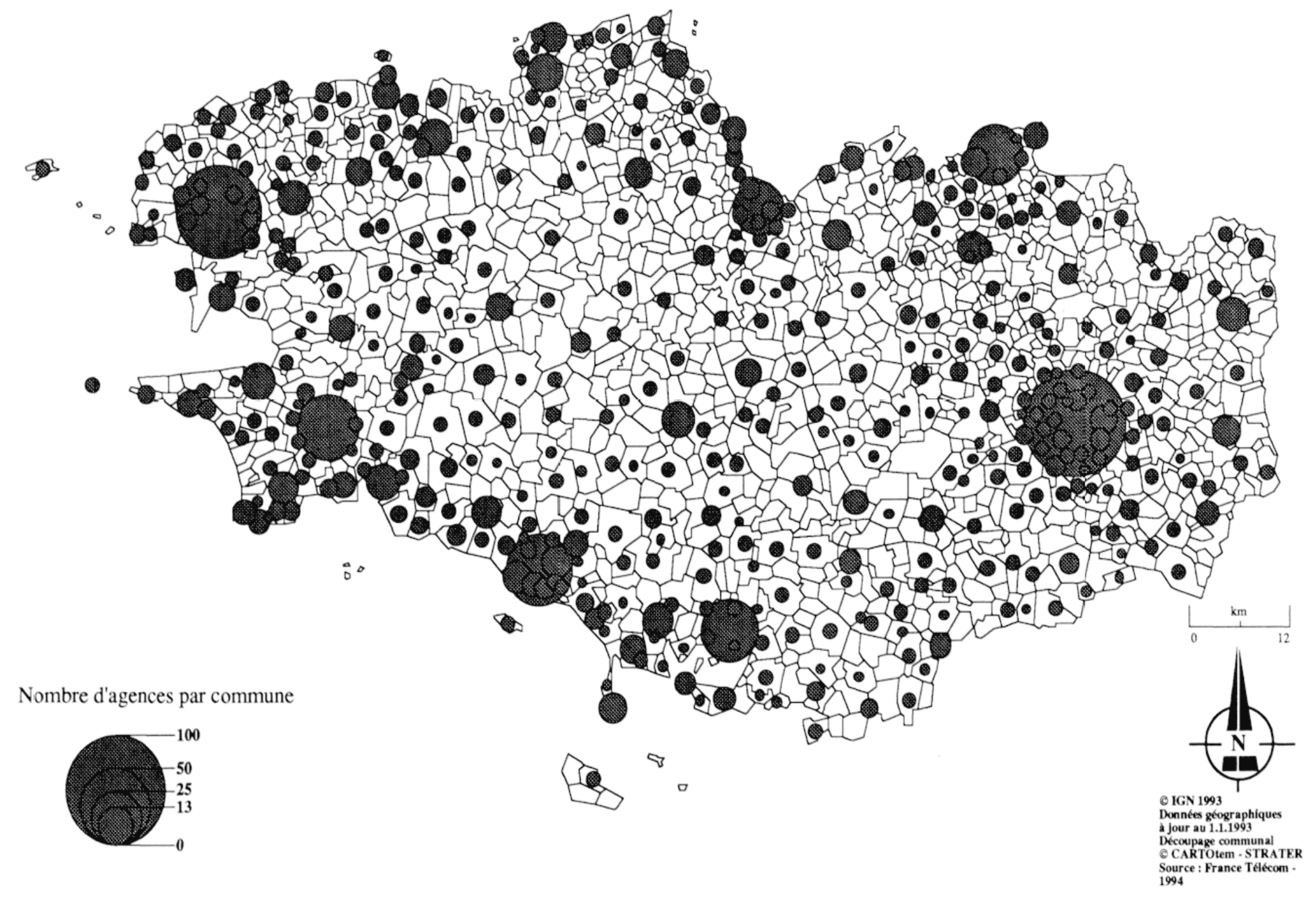


dans l'agglomération rennaise, par exemple, pour la direction régionale du Crédit Lyonnais installée sur le technopôle Rennes-Atalante à Cesson-Sévigné ou pour la Banque Populaire de l'Ouest à Montgermont. Il en va de même pour les services centraux du Crédit Mutuel de Bretagne au Relecq Kerhuon dans l'agglomération brestoise.

\section{B) DES STRATÉGIES DIFFÉRENCIÉES}

Cette première observation à partir du nombre d'agences et enseignes bancaires est très grossière. La carte est en fait le résultat de la superposition de stratégies différentes d'occupation et de maillage de l'espace. L'observation des implantations de différentes banques permet de distinguer trois types de banques :

- Les deux établissements mutualistes que sont le Crédit Agricole et le Crédit Mutuel de Bretagne ont en commun une forte densité et une très grande dispersion de leurs agences et bureaux. Ils assurent seuls la desserte des communes et bourgs ruraux tout en disposant d'un grand nombre de bureaux urbains, tant centraux que périphériques (par exemple, le CMB et le Crédit Agricole disposent respectivement de 20 et de 17 agences et bureaux à Rennes) (fig. 2).

- Les trois grandes ne sont par contre présentes que dans les villes d'une certaine importance, avec toutefois des exceptions sur le littoral (par exemple, dans les Côtes d'Armor, la BNP est présente à Perros Guirec et Saint-Cast ; la Société Générale à Erquy, Perros Guirec, Plouha, Saint-Cast, Saint-Quay ; le Crédit Lyonnais à Erquy, Perros Guirec, Saint-Quay) (fig. 3). Des banques régionales comme les deux banques populaires présentes en Bretagne - Banque populaire de l'Ouest et Banque populaire Bretagne Atlantique - ou la Banque de Bretagne apparaissent en position intermédiaire en ce sens où elles ne sont certes pas présentes dans les campagnes mais ont cependant ouvert des agences dans des bourgs et petites villes (par exemple Antrain, Bain-de-Bretagne, Combourg, Janzé, Liffré, Louvigné du Désert pour la BPO) (fig. 4).

- Le seuil d'implantation est par contre plus élevé pour les anciennes banques d'affaires et pour les institutions financières spécialisées. Les premières ne sont implantées qu'à Rennes (de Neuflize, Paribas, Worms) et Quimper (Indosuez et Paribas). Pour elles, des façades de prestige comptent plus qu'un réseau dense qui serait par nature superflu. La banque La Hénin, composante du groupe Suez spécialisée dans le financement de l'immobilier, n'est présente qu'à Rennes et fonctionne avec des correspondants dans les villes moyennes les plus éloignées de la métropole régionale (Lorient, Brest).

Les observations sur le nombre d'agences par commune et les types de localisations des différentes banques renvoient bien évidemment aux phénomènes de polarisation et à la théorie des places centrales. Cette situation de hiérarchisation des seuils d'implantation découle largement de l'histoire du système bancaire en France.

\section{C) LE POIDS DE L'HISTOIRE}

La spécialisation des banques dans le financement à court terme (banques de dépôts), les financements longs (banques de crédit), les relations avec quelques groupes industriels (hanques d'affaires) a existé jusqu'à la loi Debré de 1966 qui a libéralisé la collecte des dépôts et l'octroi des crédits. Les établissements mutualistes avaient alors déjà largement établi leur prééminence dans la collecte de l'épargne tant en milieu rural qu'en milieu urbain (où, en conséquence de l'exode 


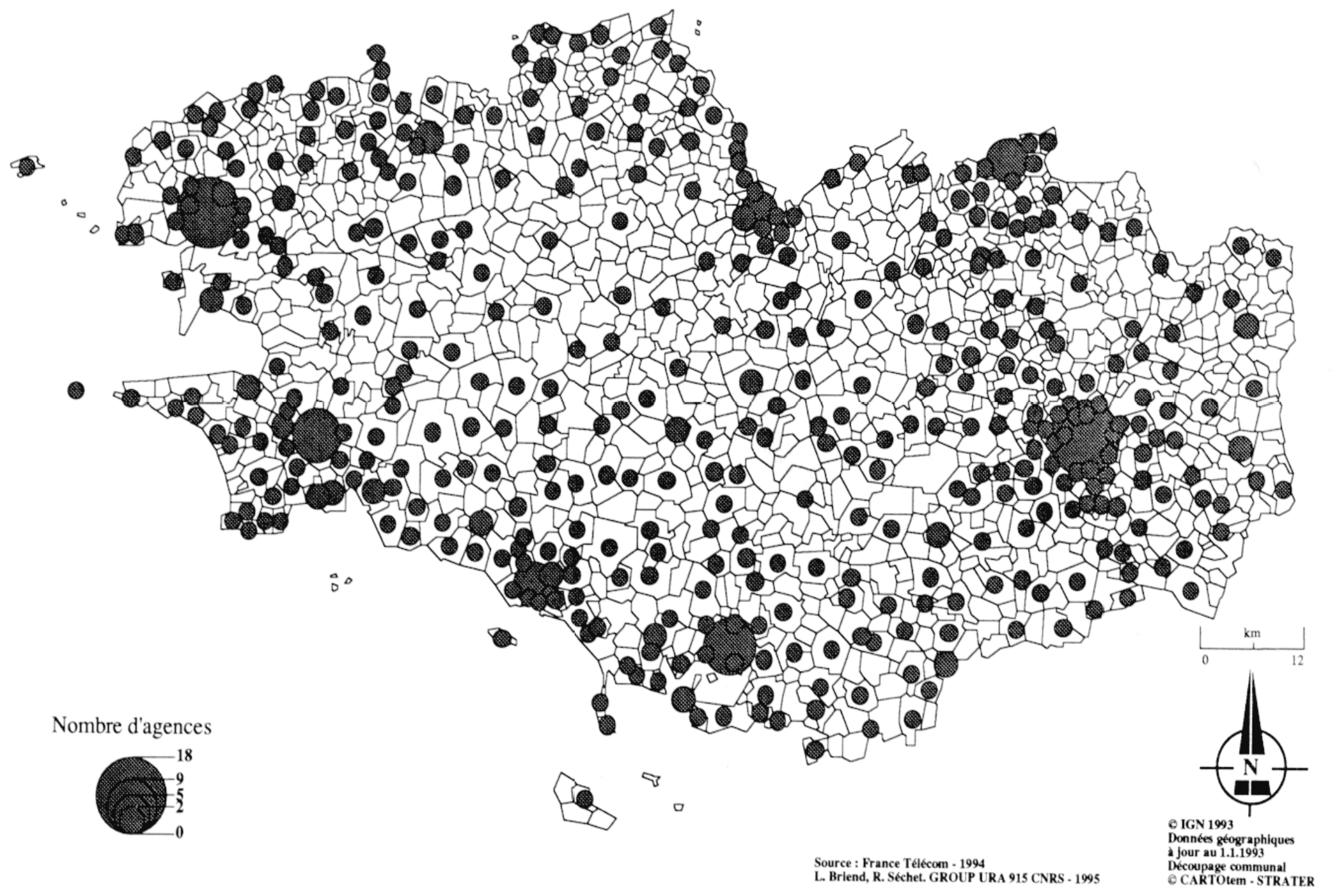

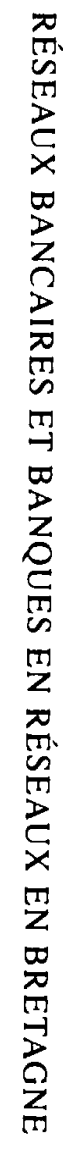

Fig. 2. - Le Crédit Agricole en Bretagne - 1994. 


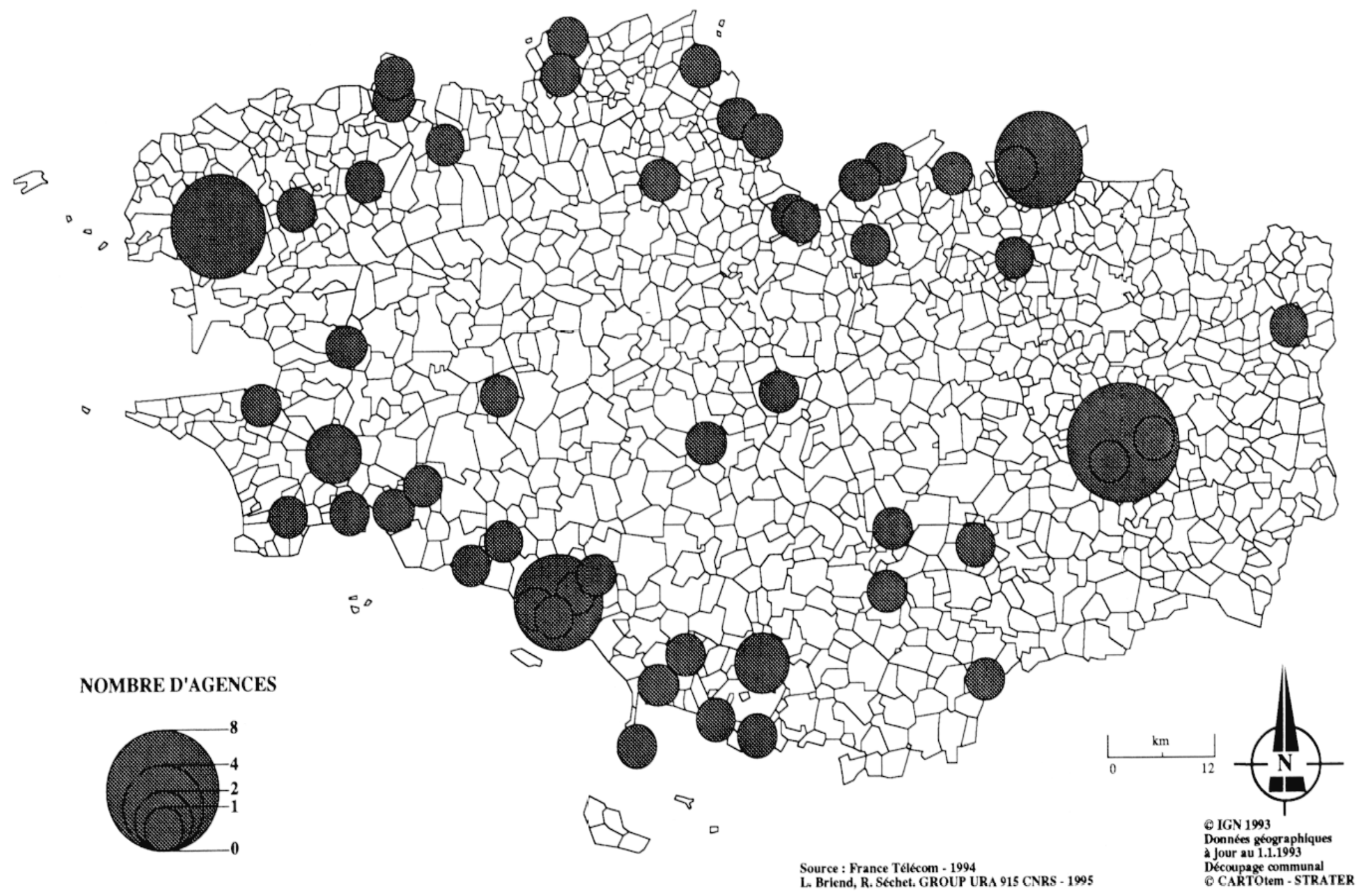

Fig. 3. - La Société Générale en Bretagne - 1994. 


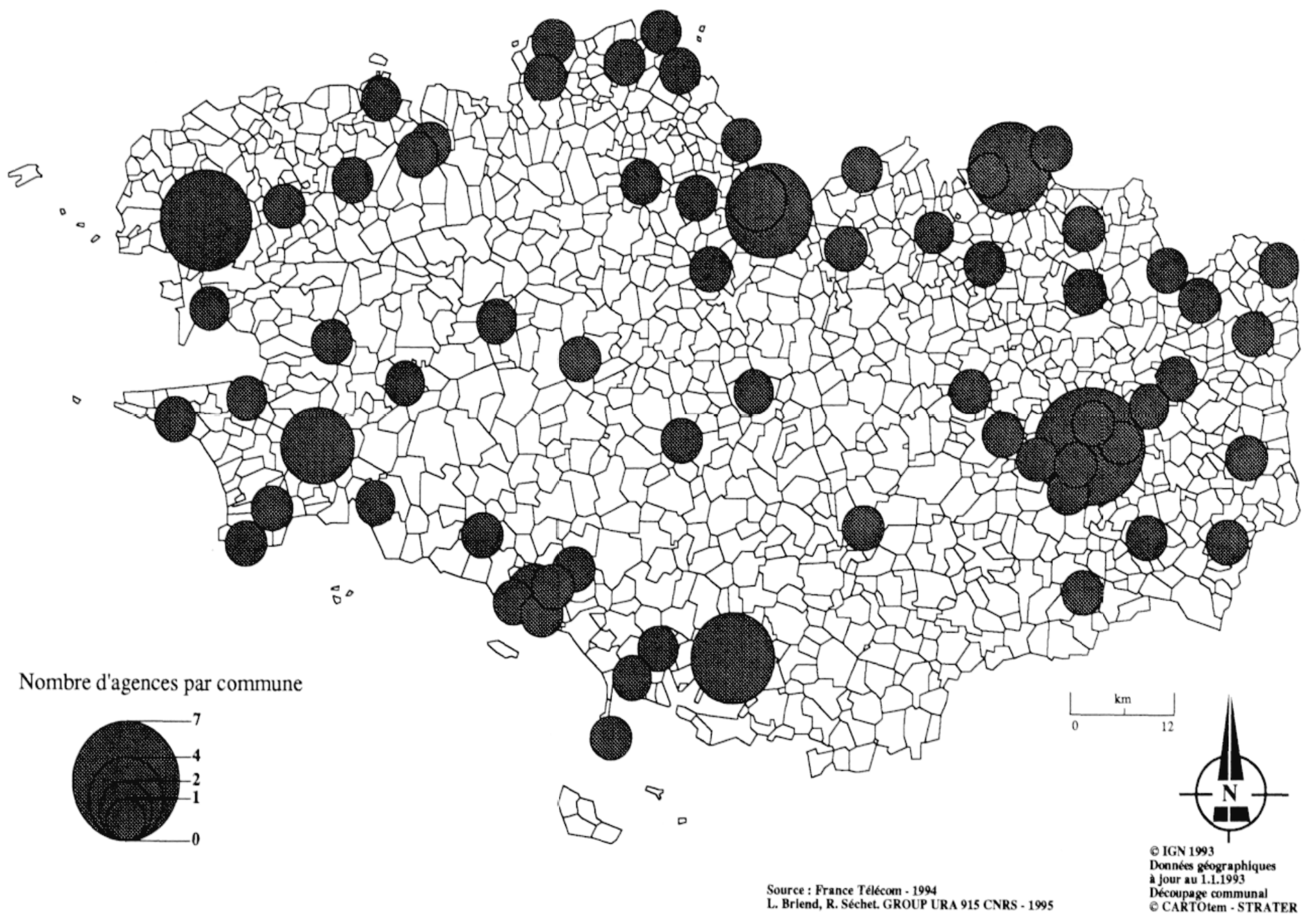

Fig. 4. - Les Banques Populaires en Bretagne - 1994. 
rural, se trouvaient un besoin de crédit mais aussi une capacité d'épargne qui allait permettre de financer la modernisation de l'agriculture). Cette occupation du terrain par les banques mutualistes était particulièrement nette en Bretagne. C'est en effet entre 1960 et 1965 que le Crédit Mutuel, né du syndicalisme agricole, notamment à Landerneau, va devenir une banque généraliste largement présente dans les quatre départements bretons.

La collecte des dépôts et des placements est une nécessité vitale pour les banques (Hommet, p. 67). En annulant l'obligation d'autorisation préalable à l'ouverture de guichets, la loi du 10 janvier 1967 a donc incité les différents établissements bancaires à se lancer dans une course poursuite pour disposer d'une assise territoriale élargie. La croissance du nombre de guichets bancaires, particulièrement rapide jusqu'en 1975, a été maximale en 1972 (1 656 ouvertures nettes pour l'ensemble du pays) (Avouyi-Dovi). La dissémination des agences dans un contexte de libéralisation n'a cependant pas mis fin à la distinction entre "banques des villes » et « banques des champs » et à l'avantage indéniable dont disposaient le Crédit Agricole et le Crédit Mutuel de Bretagne en terme de desserte de proximité.

L'existence de clientèles préférentielles qui ne sont pas réparties de manière homogène dans l'espace et ne fréquentent pas les mêmes lieux est un autre élément explicatif de la diversité topologique des réseaux d'agences bancaires. Plusieurs établissements ont assis leur renommée et leur présence dans l'espace sur la distribution des fonds d'Etat à certaines catégories socioprofessionnelles. Le Crédit Maritime est aujourd'hui encore l'archétype de la banque coopérative socioprofessionnelle. Ce n'est donc pas un hasard si, à l'exception de Rennes, toutes les agences bretonnes de cette banque dont la mission première est de soutenir la pêche (dans un contexte difficile pour la pêche, le Crédit Maritime cherche en fait sa survie en s'orientant vers la plaisance) sont situées sur le littoral (fig. 5). Les Banques Populaires, créées en vertu de la loi du 13 mars 1917 sous la forme de sociétés coopératives au bénéfice exclusif des commerçants et artisans, restent des banques urbaines; et en cela elles se distinguent fortement des autres établissements français de type mutualiste el coopératif.

La recherche d'un élargissement de leur base sociale a pu encourager certains organismes bancaires à développer des réseaux et filiales spécialisés. La CASDENBanque Populaire a ainsi été créée en 1975 : sans guichets, cette Banque Populaire nationale spécialisée dans le service aux personnels de l'Education nationale, de la Recherche et de la Culture s'appuie sur le réseau des Banques Populaires régionales. Depuis 1990 et la fin du monopole du Crédit Agricole dans l'octroi de prêts bonifiés aux agriculteurs, les Banques Populaires cherchent à devenir de véritables partenaires pour les exploitations agricoles. Là où il est puissant, le Crédit Mutuel a renforcé son influence dans le monde agricole (ainsi, en 1994, la part de marché du CMB dans la distribution des prêts bonifiés à l'agriculture en Bretagne a atteint $17 \%$ ). Traditionnellement banque de particuliers, le Crédit Mutuel a aussi développé ses activités vers les collectivités locales et les entreprises. Pour sa part, le CMB a créé en 1985 la Banque du Crédit Mutuel pour l'Entreprise (BCME), filiale spécialisée dans les prêts aux entreprises et aux collectivités publiques. Après les lois de décentralisation, ces dernières sont d'ailleurs devenues une cible pour les banques qui viennent s'attaquer aux positions du Crédit local de France (outre le CMB, la Société Générale est devenue un financier important pour les collectivités bretonnes, notamment les Départements). Les étudiants sont une autre cible des banquiers. Après des années dominées par la promotion des prêts étudiants, les banques font maintenant preuve d'imagination pour répondre à des besoins spécifiques. La Société Générale est ainsi devenue le partenaire de la SMEBA; le Crédit Lyonnais a quant à lui lancé à la rentrée universitaire 1995 l'opération STORM 


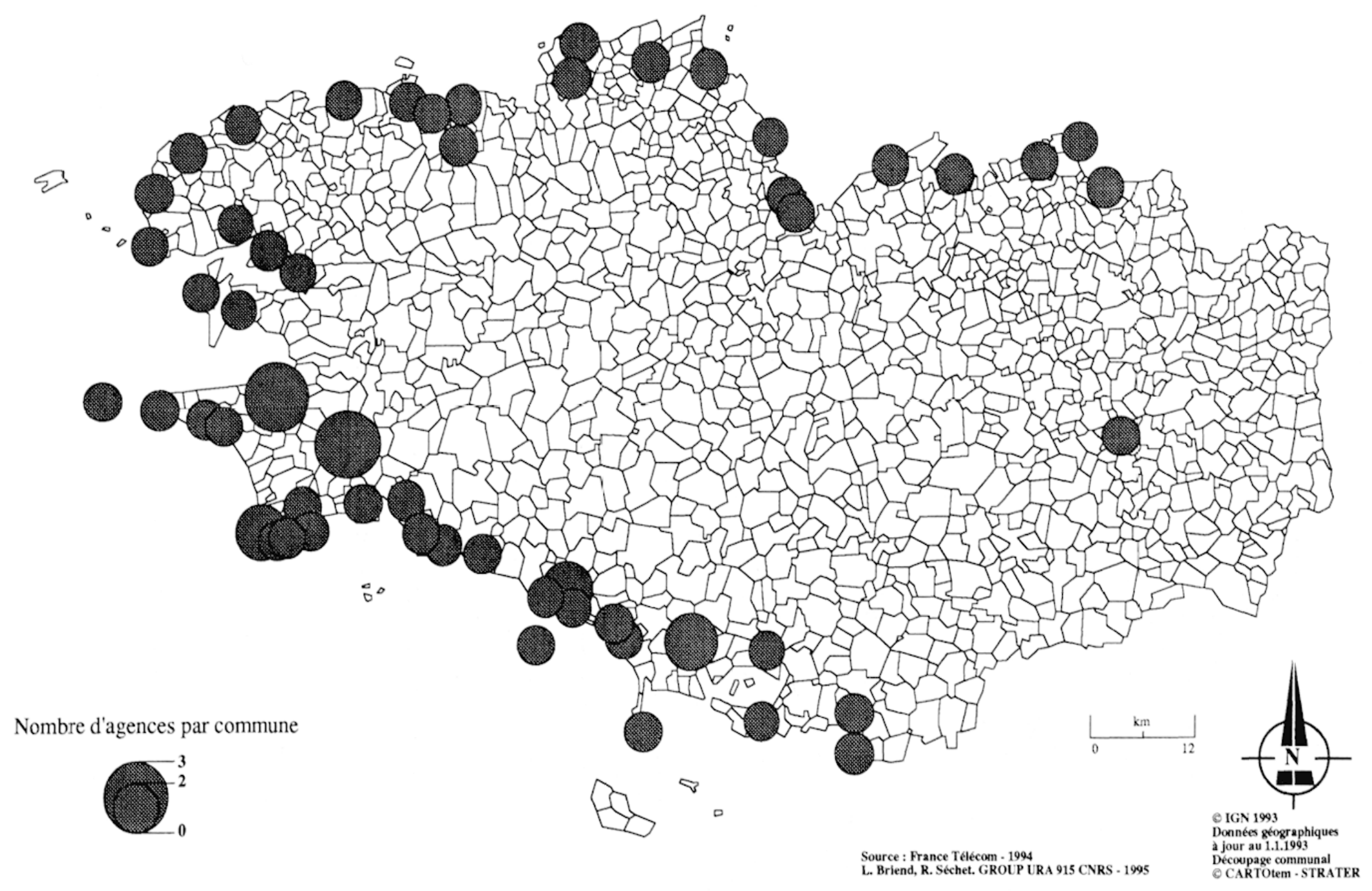

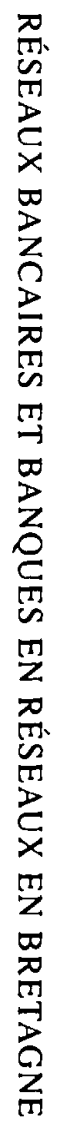

FIG. 5. - Le Crédit Maritime en Bretagne - 1994. 
(The STudents ORder More operation) pour aider les étudiants à financer leur équipement informatique ... en contrepartie de l'ouverture gratuite d'un compte. Il faut dire que les étudiants constituent pour partie le vivier de futures clientèles moyen/haut de gamme.

La composante sociologique a joué peut-être plus fortement que la répartition de la population à l'échelle régionale pour expliquer la forte densité bancaire sur le littoral breton. Plusieurs facteurs cumulent leurs effets : sur-représentation relative de groupes sociaux précocement bancarisés (artisans, commerçants, cadres) ; stratégie de captation d'une forte épargne potentielle à relier aux migrations de retraite et donc à la présence d'un grand nombre de personnes âgées ; services aux résidents en villégiature qu'il s'agit de fidéliser en les suivant dans leurs déplacements. A Perros Guirec, villégiature, vieillissement de la population (32\% des 7509 habitants ont plus de soixante ans), forte proportion de cadres (salariés de l'électronique) et d'artisans-commerçants ont joué pour aboutir au doublon trégorrois Lannion-Perros Guirec (respectivement 12 et 8 agences et bureaux).

\section{D) LA PROXIMITÉ À TEMPS PARTIEL ET EN CARTES}

La recherche d'une plus grande proximité avec le client, qui est aussi fournisseur quand il est déposant et épargnant, a produit des stratégies d'occupation de l'espace qui ont pu aboutir, grâce aux nouveaux moyens technologiques, à la mise en place de points de vente sans vendeurs.

Jouer sur les horaires d'ouvertures a pu être pour une banque un moyen d'être présente à faible coût, et se révèle être aujourd'hui un élément de la gestion stratégique des surcapacités bancaires. C'est le principe des bureaux annexes et des bureaux temporaires (c'est-à-dire des bureaux qui sont fermés au moins une journée complète sur cinq, ouverture hebdomadaire maximale autorisée en France). Le Crédit Agricole et le Crédit Mutuel de Bretagne ont largement utilisé cette formule dans leur marquage réciproque de la phase de bancarisation des années soixante aux années quatre-vingt. Dans les stations balnéaires, les horaires d'ouverturc des agences peuvent suivre un rythme saisonnier. Ainsi, l'agence de la Banque Populaire Bretagne-Atlantique de Carnac est ouverte toute la journée en été et seulement le matin en hiver.

Les guichets automatiques de banque (GAB), les distributeurs de billets (DAB) et autres « Points argent " permettent aux banques d'être là où se trouvent les gens. Les premiers véritables distributeurs de billets ont été mis en place par le Crédit agricole (carte Contact) et les Banques Populaires (Distribanque) en 1971. L'observation de listes d'agences du groupe Banques Populaires apporte des réponses à deux questions :

1) La première porte sur la localisation des sites bancaires dotés de DAB et de GAB. D'une part, ceux-ci sont plus présents dans les agences de centre-ville que dans les agences de quartiers périphériques, et, d'autre part, ils ignorent très largement le milieu rural.

2) Par ailleurs, les GAB et les points argent hors sites bancaires sont surtout installés dans les lieux de fort passage et de forte consommation d'argent (centres commerciaux et autres galeries marchandes, gares, ... mais aussi casinos). Toutefois, le Crédit Agricole a implanté des « points verts » qui permettent aux commerçants de petites communes rurales de fuurnir un service complémentaire de caissc ; quant aux possesseurs de cartes du CMB, ils peuvent effectuer des retraits d'argent dans des «Relais du Crédit Mutuel de Bretagne »c'est-à-dire, là aussi, des commerces de petites communes. 
Si l'on met à part les anciennes banques d'affaires pour lesquelles la notion de réseau ne revêt pas de réelle signification, les formes d'occupation de l'espace par les différents organismes bancaires, reflet de stratégies d'entreprises bâties sur le temps long, opposent deux grands modèles spatiaux qui correspondent pour le premier à une production dispersée et pour le second à une production concentrée. Dans la phase de bancarisation intense postérieure à la loi Debré, les différentes entreprises bancaires ont toutes recherché la proximité avec le client. La multiplication des points de vente alors même que l'informatique soulageait les tâches individuelles dans les agences a ainsi débouché sur des réseaux denses et une utilisation extensive de l'espace. Dans la phase actuelle de consolidation, pour maintenir leurs parts de marché, les banques doivent s'appuyer sur la capacité de leurs agences à capter des flux et donc optimiser localement la créativité et la force d'initiative et d'innovation de l'entreprise ou du groupe.

\section{II. - LES APPAREILS : DES BANQUES EN RÉSEAUX}

Les performances des banques dans la création de valeur ajoutée et de profit reposent sur la cohérence et l'efficacité de l'articulation entre le maillage horizontal de l'espace par les agences (dont nous avons montré l'inégale densité) et la structuration verticale des appareils en une arborescence hiérarchisée. Le passage à la polyvalence, voire à la «bancassurance », la complexité croissante des systèmes bancaires qui découle de l'interbancarité et de l'internationalisation, une concurrence accrue et une conjoncture économique qui imposent la recherche de gains de productivité ont entraîné des réorganisations internes marquées par la redéfinition des tâches de chacun des niveaux, par la montée des partenariats à tous les niveaux et par la croissance des flux d'informations et de valeur ajoutée.

\section{A) BANQUES AFB ET BANQUES COOPÉRATIVES OU L'OPPOSITION APPAREILS CENTRALISÉS / APPAREILS DÉCENTRALISÉS}

Fn terme d'organisation des appareils, la distinction entre banques AFB ct banques coopératives peut schématiquement être résumée en une distinction entre appareils centralisés, qui posent la question du rôle et de la fonction des niveaux inférieurs, et appareils décentralisés encadrés par des structures fédératives.

Les grands établissements bancaires (BNP, Crédit Lyonnais, Société Générale) ont en commun un appareil de type centralisé et une organisation hiérarchique schématiquement calquée sur les réseaux urbains : des agences presque toutes situées en milieu urbain, des succursales dotées de services spécialisés et assurant une fonction de direction de groupe dans les villes moyennes (schématiquement le niveau départemental même si cette règle est à nuancer selon les banques et en fonction des disparités de poids financier des différents départements), des directions ou délégations régionales, un siège à Paris. Cette organisation hiérarchique n'exclut pas la localisation en province de certains services. Le meilleur exemple breton est peut être fourni par le centre de comptabilité des titres de la BNP à Taden près de Dinan. Cette implantation remonte à 1938 lorsque les menaces de conflit militaire incitent la banque à transférer le plus loin possible de la frontière allemande la conservation des titres des succursales les plus menacées. Après guerre, par souci d'efficacité, l'ensemble de la conservation a été transféré à Dinan. Après la dématérialisation des titres à compter du 3 novembre 1984, l'établissement a changć dc fonction, passant de la conservation à la gestion des titres. Le centre assure aujourd'hui, d'une part, l'ensemble de la gestion comptable pour la BNP, ses filiales et les établissements qui confient la gestion de leurs portefeuilles à la BNP, et, d'autre part, la gestion fiscale pour les clients de la BNP. Le centre de Dinan est 
aujourd'hui l'un des deux centres de traitement des titres de la BNP, le second - Paris - étant orienté vers les transactions (proximité de la Bourse et des émetteurs). Ce partage des tâches production/gestion ou Paris/province apparaît somme toute classique.

Les grands réseaux mutualistes ont tous, quant à eux, une organisation comparable marquée par l'autonomie importante des niveaux intermédiaires alors que le niveau national remplit des fonctions fédératives et assure la sécurité financière du réseau. Notons que la réforme des Caisses d'épargne, entamée en 1991, a abouti à une organisation similaire (même si la nature juridique des Caisses d'épargne est bien différente de celle des établissements coopératifs).

Créées en 1917, les Banques Populaires sont aujourd'hui regroupées en une fédération qui occupe la sixième place dans le système bancaire français. Les regroupements opérationnels de banques régionales ont accompagné l'extension du réseau d'agences pour contribuer à l'ascension du groupe et à l'élargissement de ses clientèles : 600 agences et 58 banques régionales en 1938, 700 agences et 41 banques en 1966, 1400 agences et 37 banques en 1977, 1837 agences et 30 banques régionales (auxquelles s'ajoute la CASDEN-Banque Populaire) en 1995.

Le fonctionnement fédératif est réel dans le groupe des Banques Populaires. La Chambre syndicale des Banques Populaires, qui est à la fois l'association fédérative des différentes Banques populaires et leur organe de tutelle et de contrôle, est présentée comme « le Chef de réseau », chargé d'élaborer les politiques du groupe en matière de développement, d'organisation, d'informatique, de gestion des ressources humaines et de communication. Le Conseil syndical, composé du président du groupe et de neuf présidents et six directeurs généraux de banques populaires élus en assemblée générale, est le « gouvernement fédéral du groupe ». Il détermine les stratégies du groupe et prend les décisions nécessaires à son bon fonctionnement. La Caisse centrale des Banques Populaires, placée sous l'autorité directe de la Chambre syndicale, est l'organisme central bancaire et financier du groupe, garant de la sécurité collective.

Cette organisation se retrouve avec des nuances au Crédit Agricole Mutuel. La hiérarchie des caisses superpose des caisses locales; des caisses régionales dont la circonscription est, du moins en Bretagne, départementale et qui constituent le niveau de décision dans l'octroi des crédits et la gestion des fonds collectés par les caisses locales ; une caisse nationale contrôlée par les caisses régionales et qui est, selon la loi bancaire, l'organe central du Crédit Agricole. La Fédération nationale du Crédit Agricole joue avant tout un rôle en matière de communication et de représentation auprès des pouvoirs publics. Le Crédit Mutuel présente lui aussi une structure à trois niveaux. A la base, les caisses locales bénéficient d'une réelle autonomie juridique et financière. Au niveau intermédiaire, les fédérations régionales et les caisses fédérales ont des zones de compétence plus étendues que celles du Crédit Agricole puisqu'elles couvrent toujours plusieurs départements (le CMB couvre ainsi les quatre départements bretons). Au niveau national, on retrouve une Caisse centrale et une Confédération syndicale qui exerce les fonctions de représentation et d'organe central du groupe.

\section{B) LES RÉSEAUX URBAINS DANS LA RECOMPOSITION ORGANISA- TIONNELLE DES BANQUES}

Appareils centralisés et appareils décentralisés ont tous été confrontés aux mutations de l'activité bancaire et au passage de la bancarisation vers la consolidation. La redéfinition des tâches et des fonctions, les regroupements de moyens, l'établis- 
sement de partenariats avec d'autres banques et d'autres prestataires de services ont pris des formes différentes selon le type de banques. Les grandes tendances ont été un renforcement du niveau intermédiaire pour les banques AFB et une affirmation du niveau supérieur pour les banques mutualistes. Cette tendance a été moins nette pour le Crédit Agricole et le Crédit Mutuel que pour le groupe des Banques Populaires.

Le recours plus systématique des différentes Banques Populaires régionales aux services communs mis à leur disposition par la Caisse centrale permet en partie de compenser les handicaps que font peser sur la productivité des structures décentralisées et l'émiettement de la clientèle. La Caisse centrale apporte aux Banques Populaircs son concours pour les opérations de commerce international et, par ses filiales spécialisées, elle leur a permis de réaliser des économies d'échelles (Bourse, accès aux marchés financiers, gestion des titres par exemple), d'assurer la diversification des activités du groupe (assurances) et d'accroître sa présence internationale (filiales, bureaux de représentations, sociétés de services et conseils à l'export). Pour élargir encore sa gamme de produits et services diffusés par les différentes banques régionales, le groupe des Banques Populaires a signé en 1994 des accords de partenariat avec la MAAF (produits d'assurance dommages aux biens) et l'AG2R (lancement d'un produit d'assurance dépendance). C'est que l'assurance est devenue aux dires de la Chambre syndicale elle-même « le deuxième métier du Groupe Banques Populaires ".

Se présentant aussi comme la banque d'affaire des PME-PMI, le groupe des Banques Populaires a constitué le holding Banques Populaires Ingénierie SA qui a pour objectif de rationaliser et développer l'offre en direction des PME-PMI par la réunion de l'ensemble des filiales du groupe spécialisées dans l'ingénierie financière, c'est-à-dire les activités de négociation d'affaires (fusions / acquisitions) et d'accompagnement des opérations de croissance et de transmission d'entreprises ; l'ingénierie patrimoniale (gestion du patrimoine des chefs d'entreprises); l'ingénierie sociale (épargne salariale et préparation à la retraite dans le cadre de l'entreprise) ; l'ingénierie internationale (services à l'exportation). Pour favoriser les activités de commerce international, la tête de réseau du groupe a contribué à la formation d'un réseau de réseaux par la signature en 1990 d'accords de coopération avec d'autres groupes à réseaux importants, membres de la Confédération internationale du crédit populaire (dont les Volksbanken et les Raiffeisenbanken en Allemagne, la Banque Populaire Suisse et la Caisse Centrale Desjardins du Québec).

Partant d'une forte concentration (à Paris), les grandes banques ont toutes mis en cuvre dans les années soixante-dix une politique de déconcentration marquée par l'éclatement des services de production en unités d'exploitation régionales. Outre qu'elle est une nécessité fonctionnelle, c'est-à-dire une réponse à l'impossibilité de gérer en un seul lieu des effectifs nombreux, la déconcentration sur les niveaux régionaux et locaux de l'essentiel des décisions de crédit permet une plus grande proximité avec le client, une meilleure connaissance du terrain, et donc une réduction des risques. Ce processus s'est fait au profit des principales métropoles régionales. A Rennes se trouvent, par exemple, une direction régionale du Crédit Lyonnais et l'une des 13 délégations régionales de la Société Générale. Celle-ci coordonne et anime l'action des agences de Bretagne, Basse-Normandie, Pays-de-la Loire.

En ce qui concerne les banques mutualistes, un double processus contribue à renforcer tout en le hiérarchisant le niveau régional. Il s'agit d'abord des regroupements et redécoupages. Les rythmes peuvent être différents : les regroupements, qui ne sont pas nécessaires au Crédit Mutuel déjà organisé sur des bases régionales, 
sont assez lents au Crédit Agricole (à l'ouest, la dernière opération concerne le regroupement des caisses régionales de la Manche et de l'Orne) alors qu'ils ont été très rapides pour les Caisses d'épargne. En effet, le nombre de Caisses d'épargne est passé de 468 en 1983 à seulement 31 en 1995 (C. Nanterme \& Ollagnier). Cependant, le mouvement va presque toujours dans le sens d'un renforcement des pôles régionaux. Le processus est très net pour les Banques Populaires. Dans la fusion intervenue en 1988 entre la Banque Populaire d'Armorique et la Banque Populaire de l'Ouest, Rennes s'est imposée face à Saint-Brieuc (les autres regroupements intervenus depuis 1985 se sont faits au détriment de Toulon, Orléans, Montluçon, Nevers, Auxerre, Mâcon, et au profit de Nice, Tours, Clermont-Ferrand, Dijon, Besançon).

Le second processus observé au niveau régional dans l'organisation des banques mutualistes, la mise en place de services communs à plusieurs banques régionales, permet de réaliser des économies externes. Les Banques populaires privilégient aujourd'hui ce renforcement de la coopération par rapport aux fusions de banques régionales. L'informatique est un domaine classique de ces regroupements de moyens. Or les six groupements informatiques qui ont été constitués sont basés soit en région parisienne (+XALTAÏR et LIVE INFORMATIQUE) soit dans des métropoles régionales sièges de Banques Populaires (CARSO à Toulouse, CERIUS INFORMATIQUE à Dijon, Centrale de prestations de services à Metz, GC2i à Nantes). Ce dernier travaille pour les Banques Populaires du Grand Ouest (Banque Populaire Bretagne-Atlantique, Banque Populaire de l'Ouest, Banque Populaire Anjou-Vendée) et pour un certain nombre de Banques Populaires du Sud de la France (Banque Populaire de la Loire, Banque Populaire du Massif Central, Banque Populaire Provençale et Corse, Banque Populaire de Lyon). La Chambre syndicale des Banques populaires encourage aussi le partage de fonctions administratives de siège entre banques voisines (Cf. l'expérience Priam de regroupement des moyens administratifs des trois Banques populaires de La Roche-sur-Foron, Besançon et Dijon, pour la monétique et la gestion des effets et des crédits).

Le Crédit Mutuel connaît lui aussi une dynamique de regroupements de moyens destinés à partager les coûts de développement tout en maintenant l'autonomie des fédérations régionales. La BCME, également présente à Paris, n'a cessé d'étendre son aire d'intervention grâce à divers accords de partenariats conclus entre les groupes de Crédit Mutuel de Bretagne, de Loire-Atlantique - Centre-Ouest, du Massif Central, du Sud-Ouest. La fédération du Crédit Mutuel du Sud-Ouest (CMSO) et le Crédit Mutuel de Bretagne ont mis en commun leurs moyens financiers à travers une caisse fédérale commune. Le CMSO (qui, avec une soixantaine de points de vente en Gironde, Dordogne et Charente, est un petit poucet par rapport au CMB) devrait y gagner en présence pendant que le $C M B$ va trouver dans ce partenariat renforcé un terrain d'essor pour ses produits propres (assurance, produits financiers) et pour ses filiales. Le Crédit Mutuel de Bretagne a aussi créé avec le Crédit Mutuel de Loire-Atlantique et du Centre-Ouest une société, basée à Nantes, afin de concevoir des produits d'assurances pour les particuliers qui sont distribués dans les réseaux des deux groupes.

Au niveau local, l'informatisation des agences a accompagné le mouvement vers la banque universelle dans la mesure où, en allégeant les tâches d'administration et de gestion, elle a facilité la croissance des activités commerciales d'information, de conseil, de vente. La question de l'enracinement local n'est pas nouvelle pour les banques mutualistes, et tout particulièrement pour le Crédit Mutuel. A titre d'exemple, rappelons que le CMB est actionnaire de la Société d'économie mixte pour l'équipement et l'aménagement de la Bretagne et qu'il a renforcé sa présence dans les secteurs de l'économie sociale et du tourisme en milieu rural par la signa- 
ture de conventions avec l'Association de Développement de l'Insertion par l'Emploi et avec la Fédération des Pays d'accueil touristiques de Bretagne, et par la création de l'association Créavenir Bretagne chargée d'accompagner financièrement les actions en faveur du développement local. Yves Malécot, président du groupe des Banques populaires de 1963 à 1979, posait cette question de l'ancrage local lors de l'assemblée générale des Banques Populaires en 1966 : «Vous êtes les véritables banques régionales, pour trois raisons : d'abord vos éléments constitutifs, c'est-à-dire vos sociétaires, sont enracinés dans la région, ensuite vos clientèles privilégiées, les P.M.E. et les artisans, sont la trame même de l'économie de vos régions ; enfin vos dimensions sont idéales pour entretenir le contact et le dialogue [...]. Soyez les agents actifs de la politiquc nationalc d'aménagement du territoire et les animateurs du développement régional dans votre zone d'action (1). " La nouveauté est qu'elle se pose aujourd'hui à l'ensemble des banques; comme si celles-ci étaient condamnées à croiser la verticalité de leur réseaux avec l'horizontalité de mailles dont la taille serait fonction de la densité des réseaux.

Aux tâches de collecte des dépôts, de réponse aux demandes des clientèles, de gestion des crédits, les agences ont substitué ou ajouté une fonction de dynamisation du tissu économique et social local qui implique l'établissement de relations de partenariat avec les clients. L'espace local n'est plus seulement cadre neutre de l'offre de services ; il devient territoire impliqué dans la réalisation de profits et dans les objectifs stratégiques de la banque. C'est dans les entrepreneurs les plus dynamiques et les ménages solvables que se trouvent les sources potentielles de profits qui assureront la bonne santé de la banque. Il s'agit donc de les fidéliser par l'établissement de relations d'écoute et par une diversification de l'offre de produits afin de les accompagner dans leur vie et leur développement. Dans une banque universelle qui fonctionne plus sur des relations d'engagement que sur des relations à l'acte, l'entretien de l'investissement informationnel est primordial car il permet aux banques de détenir, au moins temporairement, un « monopole ex post : les firmes de bonne qualité sont « informationnellement capturées » dans la mesure où la banque qui vient de leur octroyer un crédit est à même de leur faire la meilleure proposition pour un nouveau crédit, les autres banques ne pouvant les distinguer des mauvaises » (Guille, p. 20).

Il en résulte, d'une part, une segmentation du marché du crédit en une multitude de relations de clientèles plus ou moins exclusives, et, d'autre part, une restructuration de la façon de commercialiser des produits de plus en plus ciblés. Dans un contexte de concurrence exacerbée et de bancarisation quasi complète des particuliers, l'avenir des banques dépend en partie de l'imagination de ceux qui créent et mettent en œuvre la distribution des nouveaux produits. Dans le cas des produits de base ou des produits réglementés, pour lesquels les banques proposent toutes la même chose, "l'art du marketing consiste à trouver l'astuce de présentation ou le package l'associant à d'autres produits et rendant la proposition plus attractive que celle de la concurrence » (Bernier, p. 69). Le processus a abouti à la spécialisation des points de vente structurée sur la base d'une hiérarchisation des clientèles, des prestations et des prestataires : au premier niveau, le réseau fonctionne avec des automates (DAB et $\mathrm{GAB}$ ) ; au deuxième niveau, les agences de proximité réalisent la gestion courante et le conseil standardisé ; enfin, dans les agences " haut de gamme ", sont affectés des spécialistes d'un segment de clientèle chargés de faire du conseil sur mesure (Cf. Cossalter, p. 18).

(1) En 1995, parmi leurs 441 administrateurs, les Banques Populaires comptent 219 chefs d'entreprise, 63 artisans et commerçants, 32 professions libérales. 
La redéfinition des tâches et des fonctions, les regroupements de moyens, l'établissement de partenariats avec d'autres banques et d'autres prestataires de services qui en ont découlé ont pris des formes différentes selon le type de banques mais ils sont, dans tous les cas, allés dans le sens d'une croissance des flux de valeur ajoutée et d'information et d'une interdépendance accrue entre les différents niveaux de la hiérarchie bancaire au détriment de simples relations d'autorité, de contrôle, de captation de plus-value par le sommet de la hiérarchie. Le niveau supérieur garde le monopole de l'orientation et de la définition stratégique mais le pouvoir de décision est maintenant partagé ; à l'inverse, avec l'étoffement et la structuration des services les plus spécialisés, les opérations de production et de gestion sont remontées et la fonction de gestion de la production du niveau supérieur n'a cessé de se renforcer. Autant de tendances qui traduisent l'intégration croissante des organisations bancaires.

\section{CONCLUSION : LA BANQUE, UNE ENTREPRISE-RÉSEAU}

A l'époque de bancarisation marquée par une diffusion extensive des guichets et une organisation pyramidale fait suite, après 1975, une période où les dynamiques ont moins été fonction des évolutions réglementaires que des contraintes économiques. Les choix stratégiques aboutissent dès lors à un ralentissement du rythme de croissance du nombre de guichets et à la recherche d'efficacité par l'optimisation du fonctionnement des réseaux et des modalités d'articulation entre les différents niveaux technico-organisationnels. Dans leurs grandes lignes, les évolutions de la structure (les banques en réseaux) et de l'espace (les réseaux de guichets de banques) bancaires ne sont pas sans similitudes avec celles observées dans l'industrie. Il peut donc être pertinent de reprendre à leur propos la notion d'entrepriseréseau, proposée par Robert Reich, et qui se caractérise par l'intensité des relations de partenariats externes et internalisés ; par l'intégration et le partage de valeurs communes entre concepteurs, distributeurs, clients ; par le micro-marketing et la personnalisation de l'offre.

La remise en cause d'un fonctionnement organisé selon le modèle centre-périphérie au profit d'une répartition plus équilibrée des responsabilités dans les grandes activités de production, gestion et vente, accompagnée d'une intense circulation des flux entre les différents niveaux trouve son expression spatiale dans un affinement des hiérarchisations et un triple processus d'ancrage territorial, de régionalisation, de métropolisation. Le poids de la métropole parisienne s'est renforcé en ce sens où aux activités inhérentes aux sièges sociaux (direction, définition des orientations politiques et stratégiques) s'ajoute une participation accrue à la production. C'est à ce niveau qu'a été mis en œuvre le passage vers la banque universelle et donc la diversification (par exemple, établissement de partenariats avec les compagnies d'assurances et création de filiales spécialisées dans les opérations sur titres dont la part relative dans les bilans a fortement progressé). C'est aussi à ce niveau que se gère l'insertion de la banque et de ses clients dans une économie de plus en plus mondialisée et que se négocie l'interbancarité. Le niveau intermédiaire a connu un processus de régionalisation / sélection en ce sens où les regroupements dans les banques coopératives, la mise en place de directions régionales dans les grandes banques AFB, la création de structures de gestion à forte composante partenariale se sont faites au bénéfice de capitales régionales et au détriment de villes plus petites, de rang départemental. La Bretagne n'a pas échappé à ce mouvement malgré le contrepoids que représente Brest et le CMB. L'ancrage socio-territorial (parler de territorialisation serait sans doute excessif même si les discours des banques sur leurs pratiques tendraient à accréditer ce terme) fait de l'agence un terminal de 
réseau non au sens technique de cette expression mais en référence à l'entrepriseréseau dans laquelle l'agence est le partenaire de clientèles, de collectivités locales et d'entreprises qu'il s'agit de fidéliser et de dynamiser.

Il ne faut cependant pas se méprendre sur la signification de ce passage à la banque-réseau moins clairement polarisée et dans laquelle les responsabilités sont davantage partagées. Rechercher la sécurité et limiter la prise de risque par une meilleure connaissance des clients et des contextes locaux ne favorise pas l'émergence des projets. Riccardo Petrella va plus loin dans l'analyse critique des nouvelles pratiques bancaires. Pour lui, faire de la compétitivité le but principal à court et moyen terme dans un contexte fortement concurrentiel, c'est renforcer l'affrontement pour la conquête des marchés et la défense des positions acquises. Cette logique s'empare de la logique de partenariat en ce sens où la coopération est un atout pour la compétitivité. Si les accords au sommet sont inséparables des processus de mondialisation et de globalisation des entreprises et des économies et si les regroupements régionaux relèvent de la recherche d'économies externes, force est de conclure que l'ancrage territorial des banques pourrait bien être une forme de socialisation du principe d'exclusion. En effet, l'établissement de relations d'engagement et de fidélisation qui vise en priorité les clientèles les plus rentables suppose une segmentation des clientèles au détriment des groupes, des entreprises, des espaces peu porteurs de profit. Toutefois, l'importance des réseaux mutualistes vient ici fortement atténuer une tendance dont il faudrait mesurer l'ampleur dans d'autres contextes régionaux ou internationaux.

\section{BIBLIOGRAPHIE ET SOURCES}

\section{Ouvrages et articles}

Alecian (S.), Fabre (R.), Zuccarelli (J.), 1993. - «Réseaux bancaires : la course aux guichets ", in Savy (M.) \& Veltz (P.) (dir.), Les nouveaux espaces de l'entreprise, DATAR/Editions de l'Aube, col. Monde en cours, pp. 149-162.

Association d'économie financière, 1993. - L'industrie bancaire, Revue d'économie financière, numéro spécial hiver, $563 \mathrm{p}$.

AvouYi-Dovi (S.), Boutillier (M.), 1995. - «L'évolution des banques françaises depuis le début des années quatre-vingt ", La documentation française, Problèmes économiques, $\mathrm{n}^{\circ} 2448,29$ novembre 1995, pp. 1-7 (reprise d'un texte publié dans la Lettre économique de la Caisse des Dépôts et Consignations, juin 1995).

BFRniER (J.-P.), 1995. - «Marketing particuliers : des créatifs pour produire », Banque, $\mathrm{n}^{\circ} 564$, pp. 68-70.

Burgard (J.-J.), 1191. - La banque en France, Presses de la Fondation nationale de sciences politiques, Dalloz, $395 \mathrm{p}$.

Cassou (P.-H.), 1994. - «La loi bancaire du 24 janvier 1984, un élément de la compétitivité bancaire ", Banque, $\mathrm{n}^{\circ}$ 550, pp. 22-25.

COSSALTER (C.), 1990. - Renouvellement des qualifications et de la gestion des ressources humaines dans les banques et les assurances, CEREQ, collection des études, $\mathrm{n}^{\circ} 53$, mai-juin.

Guille (M.), 1995. - « L'information du banquier, déterminant de la structure et des conditions d'équilibre du marché du crédit ", La documentation française, Problèmes économiques, $\mathrm{n}^{\circ} 2429,21$ juin 1995, pp. 19-25 (reprise de «Savoir 
bancaire spécifique, marché du crédit et intermédiation bancaire », Economie appliquée, décembre 1994).

HOMMET (J.M.), 1991. - Le système bancaire français, Dunod, 134 p.

Kerdiles (G.), 1996. - «Le Crédit mutuel de Bretagne, l'implication d'une banque régionale », Espaces, $n^{\circ} 139$, pp. 44-47.

NANTERME (P.), Ollagnier (J.-M.), 1995. - « Fusions dans le secteur financier : les règles de la réussite », Banque, $\mathrm{n}^{\circ} 564$, pp. 52-55.

Petrella (R.), 1995. - «Critique de la compétitivité », Futuribles, n 198 , pp. 71-80.

Veltz (P.), 1992. - « Hiérarchie et réseaux dans l'organisation de la production et du territoire », in BENKo (G.), LIPIETZ (A.), Les régions qui gagnent, PUF, coll. Economie en liberté, pp. 293-313.

\section{Articles de journaux}

Chaperon (I.), 1995. - « Le secteur bancaire en mal de consolidation ", Les Echos, 6 novembre.

«Le CMB s'ancre en Gironde », Ouest-France, 29 septembre 1995.

LESER (E.), 1995. - «La réforme des Caisses d'épargne renforce leur autonomie ", Le Monde, 17-18 septembre.

Luneau (D.), 1996. - «Le Crédit Mutuel crée un pôle d'assurance à Nantes », Les Echos, 21 mai.

\section{Documentations diverses}

Direction vie fédérative de la Chambre syndicale des Banques populaires, 1917 1992. 75 ans de Banques Populaires, avril 1992, 30 p.

Rapports annuels ou semestriels d'activité des Banques populaires, de la Société Générale, du Crédit Mutuel de Bretagne, de la Banque du Crédit Mutuel pour l'Entreprise. 